\title{
Recurrent Pancreatic Cystadenocarcinoma
}

National Cancer Institute

\section{Source}

National Cancer Institute. Recurrent Pancreatic Cystadenocarcinoma. NCI Thesaurus.

Code C153620.

The reemergence of pancreatic cystadenocarcinoma after a period of remission. 